\title{
Anatomic Neuroimaging Characteristics of Posterior Fossa Type A Ependymoma Subgroups
}

\author{
(D) N.D. Sabin, (D) S.N. Hwang, (DP. Klimo Jr, (D) N. Chambwe, (D) R.G. Tatevossian, (D). Patni, (D). Li, (D) F.A. Boop, (DE. Anderson,
}

(D) A. Gajjar, (D)T.E. Merchant, and (DD.W. Ellison

\begin{abstract}
BACKGROUND AND PURPOSE: Posterior fossa type A (PFA) ependymomas have 2 molecular subgroups (PFA-1 and PFA-2) and 9 subtypes. Gene expression profiling suggests that PFA-1 and PFA-2 tumors have distinct developmental origins at different rostrocaudal levels of the brainstem. We, therefore, tested the hypothesis that PFA-1 and PFA-2 ependymomas have different anatomic MR imaging characteristics at presentation.
\end{abstract}

MATERIALS AND METHODS: Two neuroradiologists reviewed the preoperative MR imaging examinations of 122 patients with PFA ependymomas and identified several anatomic characteristics, including extension through the fourth ventricular foramina and encasement of major arteries and tumor type (midfloor, roof, or lateral). Deoxyribonucleic acid methylation profiling assigned ependymomas to PFA-1 or PFA-2. Information on PFA subtype from an earlier study was also available for a subset of tumors. Associations between imaging variables and subgroup or subtype were evaluated.

RESULTS: No anatomic imaging variable was significantly associated with the PFA subgroup, but 5 PFA-2c subtype ependymomas in the cohort had a more circumscribed appearance and showed less tendency to extend through the fourth ventricular foramina or encase blood vessels, compared with other PFA subtypes.

CONCLUSIONS: PFA-1 and PFA-2 ependymomas did not have different anatomic MR imaging characteristics, and these results do not support the hypothesis that they have distinct anatomic origins. PFA-2c ependymomas appear to have a more anatomically circumscribed MR imaging appearance than the other PFA subtypes; however, this needs to be confirmed in a larger study.

ABBREVIATIONS: PF = posterior fossa; PFA = posterior fossa type A, PF-EPN-A; PFB = posterior fossa type B, PF-EPN-B

$E^{\mathrm{p}}$ pendymomas account for $>5 \%$ of pediatric CNS tumors and approximately $50 \%$ of brain tumors in children younger than 5 years of age. ${ }^{1,2}$ Approximately $90 \%$ of pediatric ependymomas are intracranial, and about $70 \%$ occur in the posterior fossa. ${ }^{3}$ Posterior fossa (PF) ependymomas are usually centered in the fourth ventricle or cerebellopontine angle cistern, often extend through the foramina of Luschka and/or the foramen of Magendie, and tend to encase cranial nerves and vascular

Received February 18, 2021; accepted after revision August 9.

From the Departments of Diagnostic Imaging (N.D.S., S.N.H., E.A.), Pathology (N.C., R.G.T., D.W.E.), Oncology (A.G.), Biostatistics (T.P., Y.L.), Radiation Oncology (T.E.M.), and Surgery (P.K., F.A.B.,), St. Jude Children's Research Hospital, Memphis,

Tennessee; and Semmes Murphey (P.K., F.A.B.), Memphis, Tennessee.

This work was supported, in part, by the National Cancer Institute through a Cancer Center Support Core Support Grant (CA21765) and by the American Lebanese Syrian Associated Charities.

Please address correspondence to Noah D. Sabin, MD, JD, Department of Diagnostic Imaging, St. Jude Children's Research Hospital, 262 Danny Thomas Place, MS-220, Memphis, TN 38105; e-mail: noah.sabin@stjude.org

-- Indicates open access to non-subscribers at www.ajnr.org

http://dx.doi.org/10.3174/ajnr.A7322 structures, making it difficult to achieve a gross total surgical resection, the most important prognostic factor for prolonged survival. ${ }^{4,5}$

Developments in molecular characterization and genetic profiling of pediatric brain tumors have led to improvements in risk stratification and associated treatment modification for these neoplasms. $^{6-8}$ For example, these methods now divide medulloblastoma into 4 molecular groups and 12 subgroups, which may be used for prediction of outcomes and stratification of treatment. ${ }^{6,8}$ Deoxyribonucleic acid methylation profiling has been used to divide $\mathrm{PF}$ ependymomas into 3 molecular groups, posterior fossa type A/PF-ependymoma (EPN)-A (PFA), posterior fossa type B/ PF-EPN-B (PFB), and posterior fossa subependymoma/PF-subependymoma. ${ }^{9-11} \mathrm{PF}$-subependymoma tumors are rare adult subependymomas, and approximately $90 \%$ of PF ependymomas are from the PFA group. ${ }^{10}$ PFA ependymomas primarily occur in infants and young children (median age at presentation, 3 years), and PFB ependymomas are mostly seen in adolescents and young adults, with a median age at presentation of 30 years. The PFA 
group is divided into 2 subgroups, PFA-1 and PFA-2. ${ }^{9}$ PFA-1 tumors express specific Homeobox family genes, which are upregulated in the caudal brainstem and upper spinal cord during CNS development, while genes located more rostrally in the developing brainstem at the midbrain-hindbrain junction (eg, EN2, CNPY1) are expressed in PFA-2 ependymomas. ${ }^{9}$ These distinct gene expression profiles suggest that PFA-1 and PFA-2 tumors originate from different brainstem locations. DNA methylation profiling has also revealed 9 subtypes of PFA ependymomas, 6 in the PFA-1 subgroup (1a through 1f) and 3 in the PFA-2 subgroup (2a through 2c). ${ }^{9}$ The PFA subgroups and subtypes are associated with different genetic and clinical variables, including different prognoses and patterns of relapse. ${ }^{9}$

The concept that imaging findings may be associated with molecularly distinct pediatric CNS tumors is supported by evidence that different molecular groups of medulloblastoma arise from different anatomic locations and that these differences are visible on MR imaging. ${ }^{12-15}$ In a recent study that extended this idea to the posterior fossa ependymoma groups, Yonezawa et $\mathrm{al}^{16}$ found that in 16 patients with posterior fossa ependymomas (9 PFAs and 7 PFBs), PFB tumors enhanced to a greater extent than PFA lesions. In that investigation, however, no significant difference was detected between PFA and PFB tumors in their tendency to extend beyond the fourth ventricle. Imaging analyses of posterior fossa ependymomas and evaluations of operative findings and techniques related to their resection have identified anatomic characteristics of the tumor that impact resectability and survival. These include location, relationship to blood vessels and cranial nerves, and extension of the tumor into adjacent anatomic regions. ${ }^{4,17-19}$

An evaluation of a small group of PFA ependymomas performed as part of a larger study of the molecular characteristics of those tumors found some differences in the anatomic locations of the PFA subgroups. ${ }^{9}$ We wanted to expand on that small combined imaging and surgical analysis by examining the differences in the anatomic characteristics seen solely on imaging between the 2 subgroups of PFA ependymomas in a larger group of patients. Our primary aim in this study was to evaluate potential differences in the anatomic characteristics of PFA-1 and PFA-2 ependymomas seen on MR imaging and determine whether those differences support different anatomic origins of the tumor subgroups. As an exploratory objective, we investigated potential different anatomic characteristics of the PFA subtypes as seen on MR imaging, with particular attention paid to the PFA-2c subtype, which is the subtype with the best prognosis. ${ }^{9}$

\section{MATERIALS AND METHODS}

The study was approved by our institutional review board. One hundred twenty-two patients with PF ependymoma treated at or who presented for consultation to the St. Jude Children's Research Hospital between 1996 and 2017 with evaluable preoperative MR imaging examinations and tumor specimens available for DNA methylation profiling were identified. The preoperative examinations were performed at outside institutions and varied with respect to pulse sequences obtained and imaging technique. Suitable studies were those that provided anatomic definition that allowed distinction of tumor from adjacent anatomic structures, including fourth ventricle foramina and major arteries. T1WI was available for all subjects; T2WI, for 120 subjects, and FLAIR images, for 106 subjects. One hundred nineteen subjects had postcontrast T1-weighted images. Two radiologists, each with Certificates of Added Qualification in neuroradiology and $>10$ years' practice experience and who were blinded to each other's evaluations, reviewed the preoperative MRIs and determined for each case whether the tumor extended below the foramen magnum, involved the foramina of Luschka, or encased the basilar artery and/or vertebral arteries. Tumors that extended below a line drawn from the tip of the clivus to the opisthion were considered to extend below the foramen magnum. Any tumor that extended beyond the margin of the fourth ventricle into one or both foramina of Luschka was characterized as involving those structures, and a tumor that appeared to extend around at least $50 \%$ of the circumference of the basilar and/or vertebral artery was classified as encasing those vessels.

The radiologists categorized the ependymomas as midfloor type, lateral type, or roof type using criteria described by U-KingIm et al. ${ }^{18}$ According to these criteria, midfloor tumors are those that generally displace the brainstem anteriorly, the entire brainstem is seen in its entirety in 1 sagittal image, and the obex is filled with tumor (Fig 1A,-B). Lateral tumors are centered in the lateral recess of the fourth ventricle or cerebellopontine angle cistern, usually do not involve the obex, and generally displace the brainstem laterally (Fig $1 C,-D$ ). For our evaluation, lesions that appeared inseparable from the roof of the fourth ventricle with a complete or partial gap between the tumor and floor were classified as roof-type tumors, though no criteria for this type of posterior fossa ependymoma were provided by U-King-Im et al (Fig $1 E,-F)$. The radiologists also evaluated the tumors for imaging evidence of necrosis, defined by the presence of centrally located nonenhancing areas within the lesions and ring enhancement. Finally, the radiologists determined whether hydrocephalus was present on the preoperative examination. Discrepancies between the radiologists' conclusions were resolved by consensus between the 2 reviewers.

Genomic DNA was extracted from 23 frozen tumor specimens following standard procedures for proteinase $\mathrm{K}$ (ThermoFisher Scientific) digestion, phenol:chloroform:isoamyl alcohol extraction and ethanol precipitation, ${ }^{20}$ and from 99 formalin-fixed and paraffin-embedded tissue specimens using the Maxwell RSC DNA formalin-fixed and paraffin-embedded kit (AS1450; Promega). DNA samples were quantified using Picogreen (Invitrogen). Genome-wide DNA methylation profiles were generated from Infinium HumanMethylation450 (450K) or MethylationEPIC BeadChip arrays (Illumina), following the manufacturer's instructions.

Ninety-nine of 122 tumors were included in our prior study and assigned to the PFA-1 or PFA-2 subgroup. ${ }^{9}$ In the present study, we first confirmed that all 122 tumors were assigned to the PFA subgroup using the Molecular Neuropathology brain tumor classifier (v11b4) (www.molecularneuropathology.org). ${ }^{21}$ Next, we classified 23 new samples as PFA-1 or PFA-2 by consensus clustering of the study cohort using the exact same parameterization as in our prior study. ${ }^{9}$ Prior subgroup designation was 

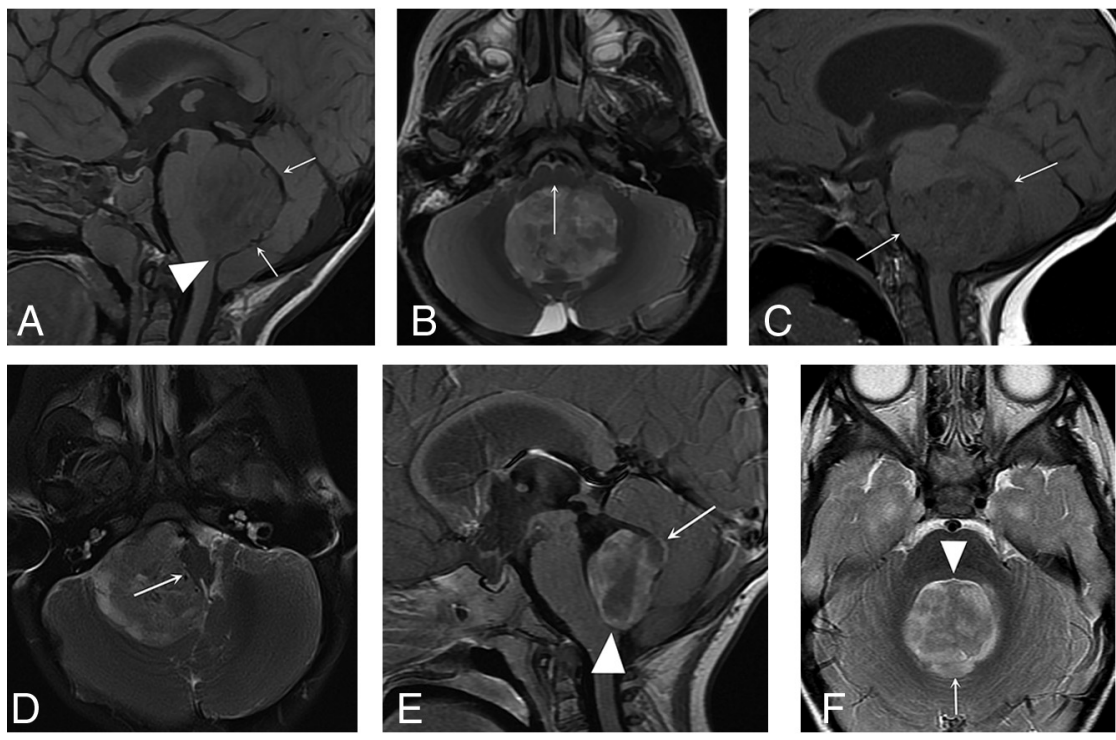

FIG 1. PFA ependymoma types based on location. Sagittal $\Pi-(A)$ and axial T2-weighted images $(B)$ : Midfloor-type posterior fossa ependymomas fill the obex (arrowhead), and a gap may be seen between the roof of the fourth ventricle and the tumor (arrows, A). The brainstem is displaced anteriorly (arrow, B), and in the sagittal plane, it is seen in its entirety in a single sagittal image. Sagittal T- (C) and axial T2-weighted images (D): Lateral-type posterior fossa ependymomas are centered in the lateral recess of the fourth ventricle or cerebellopontine angle (arrows, C) and displace the brainstem laterally (arrow, D). The brainstem usually cannot be seen in a single sagittal image. Postgadolinium sagittal $7-$ $(E)$ and axial T2-weighted images (F): Roof-type posterior fossa ependymomas appear closely associated with the roof of the fourth ventricle (arrows, $E$ and $F$ ) and do not fill the obex (arrowhead, E). A gap is usually seen between the tumor and the floor of the fourth ventricle (arrowhead, F).

consistent with the $\mathrm{k}=2$ clustering solution. New samples were labeled according to the subgroup label of their assigned consensus cluster in this joint analysis. All computational analyses were performed in $\mathrm{R}$ statistical and computing software (Version 3.6; http://www.r-project.org). Methylation array processing was performed for 122 samples as described previously, ${ }^{9}$ with the additional step of using the combineArrays function to obtain a virtual array of the subset of probes common across the $450 \mathrm{~K}$ and EPIC array platforms. Normalization and filtering steps were unchanged. Data on PFA subtypes from our prior study were available for 98 of 122 PFA ependymomas in the present study. ${ }^{9}$

Associations between imaging variables and PFA subgroup variables were investigated using the $\chi^{2}$ test because the expected cell counts were $>5$ in all contingency tables. Given the small number of tumors in some of the PFA subtypes, descriptive statistics such as frequency tables were provided to explore the relationships of the imaging characteristics of the

Table 1: Association of imaging variables with PFA ependymoma subgroups

\begin{tabular}{lrr}
\hline & Yes (\%) & No (\%) \\
\hline Extends below foramen magnum, $P=.18$ & & \\
PFA-1 & $67(89.3)$ & $8(10.7)$ \\
PFA-2 & $37(78.7)$ & $10(21.3)$ \\
Total & $104(85.2)$ & $18(14.8)$ \\
Involves foramina of Luschka, $P=.55$ & & \\
PFA-1 & $61(81.3)$ & $14(18.7)$ \\
PFA-2 & $41(87.2)$ & $6(12.8)$ \\
Total & $102(83.6)$ & $20(16.4)$ \\
Encases blood vessels, $P=.73$ & & \\
PFA-1 & $33(44.0)$ & $42(56.0)$ \\
PFA-2 & $23(48.9)$ & $24(51.1)$ \\
Total & $56(45.9)$ & $66(54.1)$ \\
Necrosis $(P=.03)^{\mathrm{a}}$ & & \\
PFA-1 & $59(80.8)$ & $14(19.2)$ \\
PFA-2 & $28(60.9)$ & $18(39.1)$ \\
Total & $87(73.1)$ & $32(26.9)$ \\
Hydrocephalus, $P=.36$ & & \\
PFA-1 & $65(86.7)$ & $10(13.3)$ \\
PFA-2 & $44(93.6)$ & $3(6.4)$ \\
Total & $109(89.3)$ & $13(10.7)$ \\
\hline
\end{tabular}

${ }^{a}$ Data for this variable were available for 119 subjects because 3 patients did not have preoperative postcontrast images available.

Table 2: Anatomic type on imaging $(P=.24)$

\begin{tabular}{lccc}
\hline & Lateral (\%) & Midfloor (\%) & Roof (\%) \\
\hline PFA-1 & $33(44.0)$ & $32(42.7)$ & $10(13.3)$ \\
PFA-2 & $14(29.8)$ & $23(48.9)$ & $10(21.3)$ \\
Total & $47(38.5)$ & $55(45.1)$ & $20(16.4)$ \\
\hline
\end{tabular}

tumors with the subtypes. All the analyses were performed in $\mathrm{R}$ software (Version 3.6.2).

\section{RESULTS}

The 122 subjects ranged in age from 5.3 months to 13.5 years (mean, 2.76 [SD, 2.26] years; median, 2.15 years), and most were male (71 [58.2\%]). Of 122 PFA ependymomas, 75 (61\%) were classified as PFA-1, and 47 (39\%), as PFA-2. There was no significant difference in the mean age (PFA-1, 2.9 years; PFA-2, 2.6 years; $P=.50$, 2-sample $t$ test) or sex distribution (PFA-1, 31 female, 44 male; PFA-2, 20 female, 27 male; $P=1.00 ; \chi^{2}$ test) between the PFA subgroups. PFA subtypes were distributed among 98 tumors for which data were available as follows: PFA1a, 13; PFA-1b, 16; PFA-1c, 4; PFA-1d, 9; PFA-1e, 14; PFA-1f, 4; PFA-2a, 24; PFA-2b, 9; PFA-2c, 5.

None of the imaging variables were associated with the 2 PFA subgroups except for necrosis (Tables 1 and 2). Review of contingency tables showed a larger proportion of tumors of the PFA-2c subtype not extending below the foramen magnum, not involving the foramina of Luschka, and not encasing vessels compared with the other PFA-2 subtypes or all other subtypes taken together (Tables 3 and 4). A greater proportion of PFA-2c ependymomas were of the midfloor type than the other PFA-2 subtypes or all other subtypes taken together. On the basis of these findings, a flag variable that incorporated the variables of "no" for extension below the foramen magnum and "no" for encasement of vessels and midfloor-type was used, and a frequency table showed that a much greater proportion of the PFA-2c ependymomas had the 
Table 3: Imaging characteristics of PFA-2c ependymomas compared with other PFA-2 subtypes and all other PFA subtypes ${ }^{a}$

\begin{tabular}{lcc}
\hline & Yes & No \\
\hline Extends below foramen magnum & & \\
PFA-2c & $0(0 \%)$ & $5(100 \%)$ \\
PFA-2a and PFA-2b & $29(87.9 \%)$ & $4(12.1 \%)$ \\
All other PFA tumors & $96(88.9 \%)$ & $12(11.1 \%)$ \\
Encases blood vessels & & \\
PFA-2c & $0(0 \%)$ & $5(100 \%)$ \\
PFA-2a and PFA-2b & $17(51.5 \%)$ & $16(48.5 \%)$ \\
All other PFA tumors & $50(46.3 \%)$ & $58(53.7 \%)$ \\
Involves foramina of Luschka & & \\
PFA-2c & $2(40 \%)$ & $3(60 \%)$ \\
PFA-2a and PFA-2b & $31(93.9 \%)$ & $2(6.1 \%)$ \\
All other PFA tumors & $92(85.2 \%)$ & $16(14.8 \%)$ \\
\hline
\end{tabular}

aninety-eight subjects had subtype data available. "All other PFA tumors" includes the PFA-1 subgroup and the PFA-2a and $2 b$ subtypes. The 15 subjects with PFA-1 subgroup ependymomas that could not be subtyped were included in the category of All other PFA tumors. The 8 patients with PFA-2 tumors that could not be subtyped were not included in this analysis.

Table 4: Imaging type of PFA-2c ependymomas compared with other PFA-2 subtypes and all other PFA subtypes ${ }^{a}$

\begin{tabular}{lccc}
\hline & Lateral & Midfloor & Roof \\
\hline PFA-2c & $0(0 \%)$ & $5(100 \%)$ & $0(0 \%)$ \\
PFA-2a and PFA-2b & $10(30.3 \%)$ & $14(42.4 \%)$ & $9(27.3 \%)$ \\
All other PFA tumors & $43(39.8 \%)$ & $46(42.6 \%)$ & $19(17.6 \%)$ \\
\hline
\end{tabular}

a Ninety-eight subjects had subtype data available. "All other PFA tumors" includes the PFA-1 subgroup and the PFA- $2 a$ and $2 b$ subtypes. The 15 subjects with PFA-1 subgroup ependymomas that could not be subtyped were included in the category of All other PFA tumors. The 8 patients with PFA-2 tumors that could not be subtyped were not included in this analysis.

Table 5: PFA-2c ependymomas compared with other PFA-2 ependymoma subtypes and all other PFA subtypes with respect to flag variable $1^{\mathrm{a}}$

\begin{tabular}{llc}
\hline \multicolumn{1}{c}{${\text { Flag Variable } 1^{\mathrm{b}}}$} & \multicolumn{1}{c}{ Yes } & No \\
\hline PFA-2c & $5(100 \%)$ & $0(0 \%)$ \\
PFA-2a and PFA-2b & $3(9 \%)$ & $30(91 \%)$ \\
All other PFA tumors & $6(5.6 \%)$ & $102(94.4 \%)$ \\
\hline
\end{tabular}

a Ninety-eight subjects had subtype data available. "All other PFA tumors" includes the PFA-1 subgroup and the PFA-2a and $2 b$ subtypes. The 15 subjects with PFA-1 subgroup ependymomas that could not be subtyped were included in the category of All other PFA tumors. The 9 patients with PFA-2 tumors that could not be subtyped were not included in this analysis.

b The flag variable incorporates the following 3 variables: "no" for extension below the foramen magnum, "no" for encases blood vessels, and "midfloor-type" tumor.

features of the flag variable than other PFA-2 subtypes or all the other subtypes as a whole (Table 5).

\section{DISCUSSION}

Previous investigations of PF ependymomas have revealed associations between tumor location and operative or disease outcomes. ${ }^{4,5,17,18,22,23}$ For example, Ikezaki et al, ${ }^{17}$ U-King-Im et al, ${ }^{18}$ and Nagib and O'Fallon ${ }^{22}$ noted lower survival rates and greater difficulty in obtaining a gross total resection for laterally located tumors (those involving the lateral recess of the fourth ventricle and cerebellopontine angle). Sanford et $\mathrm{al}^{5}$ pointed out that cerebellopontine angle ependymomas in children arising from the foramen of Luschka and the lateral aspect of the medulla tend to encase cranial nerves and blood vessels and are difficult to resect completely, leading to lower survival rates. Sabin et $\mathrm{al}^{4}$ determined that pediatric $\mathrm{PF}$ ependymomas could be divided into 2 distinct anatomic groups based on the location of their centroids and that the groups were associated with differences in progression-free survival and centralversus-lateral tumor location. The results of these studies with respect to clinical outcomes of posterior fossa ependymomas contributed to our hypothesis that the anatomic characteristics of a pediatric PFA ependymoma might also help predict its subgroup. In fact, though no survival difference was shown among the subgroups, an imaging and operative analysis of 40 tumors described in the article by Pajtler et $\mathrm{al}^{9}$ outlining the PFA ependymoma subgroups and subtypes found that the PFA-1 lesions were more likely to be located laterally than the PFA-2 subgroup and that the origin of PFA-2 tumors was more frequently from the roof of the fourth ventricle than the origin of PFA-1 ependymomas, suggesting that the subgroups arise from cells at different brainstem levels. These prior investigations suggested that an imaging evaluation of a larger group of PFA ependymomas would result in identification of distinct anatomic markers for the PFA subgroups.

In this investigation, however, traditional anatomic characteristics seen on imaging could not be used to separate PFA tumors into their subgroups, and the findings do not provide support for the hypothesis that the PFA-1 and PFA-2 subgroups arise in different locations. The association of necrosis with subgroups is of uncertain significance. If different sites of origin for the PFA subgroups lead to different vascular supplies for the PFA-1 and PFA-2 tumors, this difference could, theoretically, cause the PFA-1 subgroup to have more tenuous neovascularization with a greater tendency to thrombosis and necrosis. Our results, however, suggest that gross anatomic characteristics of PFA ependymomas visible on MR imaging at presentation are not indicative of where PFA-1 and PFA-2 tumors arise. This outcome may reflect loss of visibility on MR imaging of key anatomic distinctions related to the PFA subgroups by the time a tumor has grown large enough to cause symptoms. Given their ability to capture more subtle differences between imaging characteristics of tumors, machine learning techniques and radiomics may be beneficial in future efforts to use imaging to classify PFA ependymomas by subgroup and determine their anatomic origins. These imaging approaches have recently been used with MR imaging to predict the subgroups of medulloblastomas. ${ }^{24,25}$

Examination of the imaging characteristics of tumors among the PFA subtypes, while limited by the small number of subjects in some of the subtypes, revealed that a larger proportion of PFA$2 c$ ependymomas did not involve fourth ventricle foramina and/ or did not demonstrate vascular encasement compared with other subtypes. A greater proportion of PFA-2c tumors also showed a group of imaging characteristics contained in a flag variable than the other PFA subtypes. These variables (no extension below the foramen magnum, no encasement of vessels, and midfloor-type) suggest that the PFA-2c lesions tend to be more anatomically circumscribed than PFA ependymomas of other subtypes. PFA-2c tumors had the best survival profile of all the PFA subtypes in our earlier study, ${ }^{9}$ and this could be related to a greater proportion of that subtype having imaging characteristics, contained in the flag variable, associated with greater resectability and better outcomes. ${ }^{4,5,18,19,22,23}$ Although additional study of larger numbers of PFA-2c subtype ependymomas is needed 

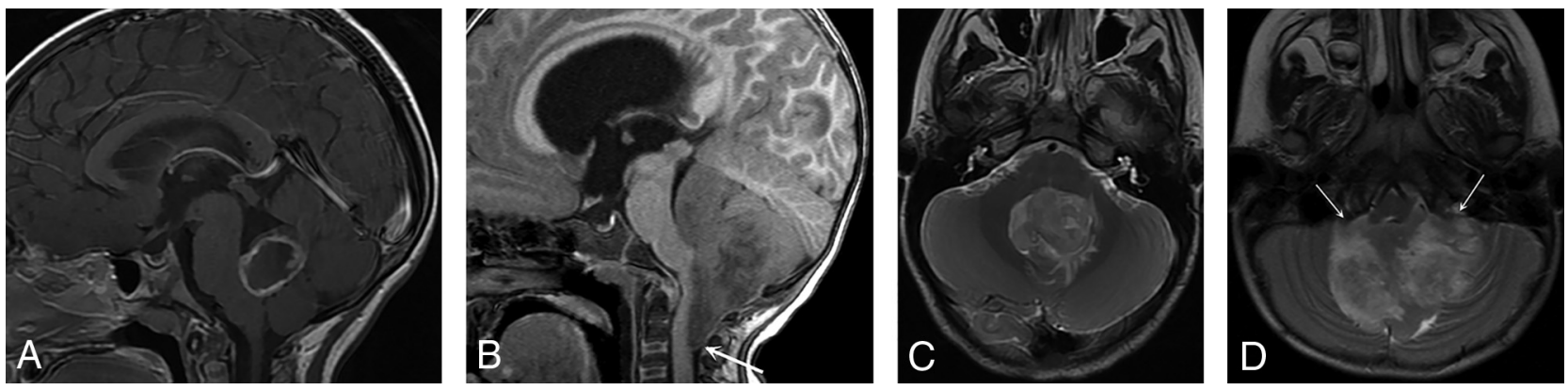

FIG 2. PFA-2c ependymoma versus another subtype. Postgadolinium sagittal T1- (A) and axial T2-weighted (C) MR images of a PFA-2c subtype ependymoma and sagittal T7- $(B)$ and axial T2-weighted (D) MR images of another PFA ependymoma subtype (1b) show that the PFA-2c ependymoma has a more circumscribed appearance in that it does not wrap around the brainstem or extend below the foramen magnum in distinction from the PFA-lb subtype (arrow, B). The PFA-2c ependymoma in this case did not extend through the foramina of Luschka, unlike the PFA-lb tumor (arrows, D), or encase the basilar or vertebral arteries. This more circumscribed appearance was seen in the 5 PFA-2c tumors in our study, though evaluation of a larger number of this subtype is needed to confirm this appearance as a characteristic of PFA-2c ependymomas.

before any firm conclusions can be made, the subtype appears to have a distinct appearance that may be associated with its better outcome compared with the other PFA subtypes (Fig 2). If accurate imaging prediction of the PFA-2c subtype can be achieved, it would provide a noninvasive method to aid in clinical decisionmaking and determining prognosis.

Limitations of this investigation include the heterogeneity of the preoperative MR imaging examinations, which were performed at different institutions and with inconsistent imaging techniques. This might have limited consistent evaluation of the tumors, including determination of anatomic areas of involvement. The studies were only included, however, if they provided adequate visualization of the posterior fossa structures of interest, and all determinations of tumor characteristics were made by 2 neuroradiologists, with any differences resolved by consensus. Another limitation was the difficulty in distinguishing midfloor-type tumors from rooftype tumors because both types are centered in the fourth ventricle and it was sometimes difficult to determine whether a gap between the tumor and roof or floor of the fourth ventricle was present. Thus, some tumors may have been misclassified as midfloor or roof type. This limitation may have been lessened by the use of consensus between the neuroradiologists for difficult cases.

\section{CONCLUSIONS}

Pediatric PFA ependymomas cannot be separated into their subgroups on the basis of traditional MR imaging characteristics, and these imaging characteristics, by themselves, do not provide support for different sites of origin. Evaluation of limited numbers of PFA ependymoma subtypes suggests that the PFA-2c subtype may have distinctive imaging characteristics; however, this possibility needs to be confirmed using a suitable statistical method with a larger number of PFA subtypes.

\section{ACKNOWLEDGMENTS}

The authors thank Dr Zoltan Patay for his review of the manuscript and Ms Mary Baltimore for her assistance in its preparation.

Disclosures: Noah D. Sabin—RELATED: Grant: National Cancer Institute, Comments: Cancer Center Support Core Support Grant (CA21765).* Amar Gajjar-UNRELATED: Consultancy: Genentech/Roche. *Money paid to the institution.

\section{REFERENCES}

1. Ostrom QT, Patil N, Cioffi G, et al. CBTRUS Statistical Report: Primary Brain and Other Central Nervous System Tumors Diagnosed in the United States in 2013-2017. Neuro Oncol 2020;22: iv1-96 CrossRef Medline

2. Zhang C, Ostrom QT, Semmes EC, et al; Glioma International CaseControl Study (GICC). Genetic predisposition to longer telomere length and risk of childhood, adolescent and adult-onset ependymoma. Acta Neuropathol Commun 2020;8:173 CrossRef Medline

3. Vaidya K, Smee R, Williams JR. Prognostic factors and treatment options for paediatric ependymomas. J Clin Neurosci 2012;19:122835 CrossRef Medline

4. Sabin ND, Merchant TE, Li X, et al. Quantitative imaging analysis of posterior fossa ependymoma location in children. Childs Nerv Syst 2016;32:1441-47 CrossRef Medline

5. Sanford RA, Merchant TE, Zwienenberg-Lee M, et al. Advances in surgical techniques for resection of childhood cerebellopontine angle ependymomas are key to survival. Childs Nerv Syst 2009;25:1229-40 CrossRef Medline

6. Gajjar A, Robinson GW, Smith KS, et al. Outcomes by clinical and molecular features in children with medulloblastoma treated with risk-adapted therapy: results of an International Phase III Trial (SJMB03). J Clin Oncol 2021;39:822-35 CrossRef Medline

7. Juraschka K, Taylor MD. Medulloblastoma in the age of molecular subgroups: a review. J Neurosurg Pediatr 2019;24:353-63 CrossRef Medline

8. Malbari F, Lindsay H. Genetics of common pediatric brain tumors. Pediatr Neurol 2020;104:3-12 CrossRef Medline

9. Pajtler KW, Wen J, Sill M, et al. Molecular heterogeneity and CXorf67 alterations in posterior fossa group A (PFA) ependymomas. Acta Neuropathol 2018;136:211-26 CrossRef Medline

10. Pajtler KW, Witt H, Sill M, et al. Molecular classification of ependymal tumors across all CNS compartments, histopathological grades, and age groups. Cancer Cell 2015;27:728-43 CrossRef Medline

11. Louis DN, Perry A, Wesseling P, et al. The 2021 WHO Classification of Tumors of the Central Nervous System: a summary. Neuro Oncol 2021;23:1231-51 CrossRef Medline

12. Gibson P, Tong Y, Robinson G, et al. Subtypes of medulloblastoma have distinct developmental origins. Nature 2010;468:1095-99 CrossRef Medline

13. Ly KI, Wen PY, Huang RY. Imaging of central nervous system tumors based on the $\mathbf{2 0 1 6}$ World Health Organization Classification. Neurol Clin 2020;38:95-113 CrossRef Medline

14. Patay Z, DeSain LA, Hwang SN, et al. MR imaging characteristics of wingless-type-subgroup pediatric medulloblastoma. AJNR Am J Neuroradiol 2015;36:2386-93 CrossRef Medline 
15. Perreault S, Ramaswamy V, Achrol AS, et al. MRI surrogates for molecular subgroups of medulloblastoma. AJNR Am J Neuroradiol 2014;35:1263-69 CrossRef Medline

16. Yonezawa U, Karlowee V, Amatya VJ, et al. Radiology profile as a potential instrument to differentiate between posterior fossa ependymoma (PF-EPN) group A and B. World Neurosurg 2020;140: e320-27 CrossRef Medline

17. Ikezaki K, Matsushima T, Inoue T, et al. Correlation of microanatomical localization with postoperative survival in posterior fossa ependymomas. Neurosurgery 1993;32:38-44 CrossRef Medline

18. U-King-Im JM, Taylor MD, Raybaud C. Posterior fossa ependymomas: new radiological classification with surgical correlation. Childs Nerv Syst 2010;26:1765-72 CrossRef Medline

19. Ma SC, Li CD, Agazzi S, et al. Clinical characteristics and prognostic factors of treatment in pediatric posterior cranial fossa ependymoma. Pediatr Neurosurg 2019;54:98-107 CrossRef Medline
20. Pearson H, Stirling D. DNA extraction from tissue. Methods Mol Biol 2003;226:33-4 CrossRef Medline

21. Capper D, Jones DTW, Sill M, et al. DNA methylation-based classification of central nervous system tumours. Nature 2018;555:46974 CrossRef Medline

22. Nagib MG, O'Fallon MT. Posterior fossa lateral ependymoma in childhood. Pediatr Neurosurg 1996;24:299-305 CrossRef Medline

23. Witt H, Mack SC, Ryzhova M, et al. Delineation of two clinically and molecularly distinct subgroups of posterior fossa ependymoma. Cancer Cell 2011;20:143-57 CrossRef Medline

24. Iv M, Zhou M, Shpanskaya K, et al. MR imaging-based radiomic signatures of distinct molecular subgroups of medulloblastoma. AJNR Am J Neuroradiol 2019;40:154-61 CrossRef Medline

25. Yan J, Liu L, Wang W, et al. Radiomic features from multi-parameter MRI combined with clinical parameters predict molecular subgroups in patients with medulloblastoma. Front Oncol 2020;10:558162 CrossRef Medline 